\title{
Introduction to Actors, Agents, and Avatars: Visualizing Digital Humans in E-Commerce and Social Media Minitrack
}

\author{
Mike Seymour \\ The University of Sydney \\ mike.seymour@sydney.edu.au
}

\author{
Lingyao (Ivy) Yuan \\ Iowa State University \\ 1yuan@iastate.edu
}

\author{
Kai Riemer \\ The University of Sydney \\ Kai.riemer@sydney.edu.au
}

Agents, and Avatars: Visualizing Digital Humans in E-Commerce and Social Media minitrack focuses on the visualization and application of digital human characters in the context of E-commerce and social media disciplines. As visualization technology advances in recent years, digital humans have been transforming the way we work, live, play, and learn. Realistic digital humans have been deployed in multiple areas, including e-commerce and social media. In social media, human realistic digital agents have become virtual influencers. In electronic commerce, virtual agents have been deployed as digital sales assistants, fashion advisers, financial consultants and personal shoppers. In particular, digital humans will become more relevant as social media is further integrated into the retail customer experience.

Comparing with other mini-tracks on artificial intelligence at HICSS, this mini track focuses on the visualization aspect of digital human in particular. We welcome submissions on any technology advancement to create human-realistic avatars and agents. This mini-track also emphasizes the importance of investigating the behavioral and perception outcomes, uncovering the complicated interactive nature among the technology, the end user, the tasks, as well as the organizational environment, and opening the black box of the underlying cognitive processes undertaken by the end user while interacting with digital humans. The adoption and use of digital humans can provide incomparable guidance to the creation of such digital characters. We hope this mini-track bridges academic research with practitioner concerns to create synergy on promoting the creation and usage of digital humans.

With the strong support of the HICSS Internet and the Digital Economy Track chairs as well as the contributing authors and quality reviewers, we have launched this minitrack for the first time this year. We positioned this minitrack as a place for researchers or practitioners from diverse background to share their research and ideas. This year, Actors, Agents, and Avatars: Visualizing Digital Humans in
E-Commerce and Social Media minitrack has accepted two research papers. Both papers have strong theoretical foundations as well as great practical implications, satisfying our expectation of using this mini-track as a platform to bridging academic research with practical inspirations.

The paper, Help! I Have a Problem" Differences between a Humanlike and Robot-like Chatbot Avatar in Complaint Management, by Anne Fota, Katja Wagner, Tobias Roeding, and Hanna Schramm-Klein, focuses using chatbot for after-sales customer service, particularly services handling customer complaints. As part of the chatbot features, the authors examine whether the chatbot avatar resembles a human, whether the chatbot is empathetic, and whether compensation is given to the customer. The authors find that the human-like avatar choice, empathetic reaction, and monetary compensation play a decisive role in influencing user behavior, increasing the probability that the customer, despite a complaint, returns and buys again from the retailer. The manuscript has great practical implication for companying that are interested in deploying chatbot to provide customer services and post-sales services.

The paper, Face It, Users Don't Care: Affinity and Trustworthiness of Imperfect Digital Humans, by Mike seymour, Lingyao Yuan, Alan Dennis, and Kai Riemer, examined individuals' tolerance of visual imperfection of digital human faces. As the authors raised, humans are very sensitive to the slightest imperfections in the visual design of artificial agents. Therefore, pursuing photorealistic avatars or human agents that are indistinguishable from real people has been the goal of many visual design companies. However, there lacks concrete scientific evidence to prove that the recognition of such imperfection would for sure lead to negative behavior or perception consequences, such as lower acceptance, less effectiveness or even aversion. How much would people tolerate visual imperfection on digital agents? The answer to this question not only tests the applicability of the Uncanny Valley Theory to the visual design of digital avatars, but also carries great 
value to practitioners who are creating avatars and human realistic agents. The results reveal that participants indeed noticed the video imperfections, but this did not adversely affect their behavior or perception. Once digital humans become close to realistic, users simply do not care about visual imperfections. 